CWRU-P6-97

CERN-TH-97/122

astro-ph/9706227

\title{
THE END OF THE AGE PROBLEM, AND THE CASE FOR A COSMOLOGICAL CONSTANT REVISITED
}

\author{
Lawrence M. Krauss ${ }^{1,2}$ \\ ${ }^{1}$ Theory Division, CERN, CH-1211, Geneva 23, Switzerland \\ ${ }^{2}$ Departments of Physics and Astronomy \\ Case Western Reserve University \\ Cleveland, $\mathrm{OH}$ 44106-7079 \\ (submitted to Science)
}

\begin{abstract}
The lower limit on the age of the universe derived from globular cluster dating techniques, which previously strongly motivated a non-zero cosmological constant, has now been dramatically reduced, allowing consistency for a flat matter dominated universe with a Hubble Constant, $H_{0} \leq 66 \mathrm{kms}^{-1} \mathrm{Mpc}^{-1}$. The case for an open universe versus a flat universe with non-zero cosmological constant is reanalyzed in this context, incorporating not only the new age data, but also updates on baryon abundance constraints, and large scale structure arguments. For the first time, the allowed parameter space for the density of non-relativistic matter appears larger for an open universe than for a flat universe with cosmological constant, while a flat universe with zero cosmological constant remains strongly disfavored. Several other preliminary observations suggest a non-zero cosmological constant, but a definitive determination awaits refined measurements of $q_{0}$, and small scale anisotropies of the Cosmic Microwave background. I argue that fundamental theoretical arguments favor a non-zero cosmological constant over an open universe. However, if either case is confirmed, the challenges posed for fundamental particle physics will be great.
\end{abstract}


The cosmological model perhaps most strongly favored by the data over the past few years has involved a proposal which is heretical from an elementary particle physics perspective. In order to reconcile a flat universe - favored by both inflationary models and by the longstanding flatness problem in cosmology - with the apparent age of globular clusters, and the fact that many estimates for the clustered mass density on large scales suggest that insufficient non-relativistic matter exists to achieve a flat universe, the idea that the cosmological constant is non-zero has been invoked[1]. Most recently the inclusion of additional arguments associated with the baryon density of the Universe and large scale structure have further strengthened the case for a cosmological constant [2, [3].

The problem with this from a fundamental perspective is that a cosmological constant-associated in modern parlance with a non-zero vacuum energy density in the universe - on a scale which would be cosmologically relevant and yet still allowed today would take a value which is roughly 125 orders of magnitude smaller than the naive value one might expect based on considerations of quantum mechanics and gravity (see for example [4]). This apparent discrepancy would involve the most extreme fine tuning problem known in physics, and for this reason many particle physicists would prefer any mechanism which would drive the cosmological constant to be exactly zero today.

The possibility that cosmology might force physicists to have to directly confront this longstanding issue in fundamental physics is exciting, but at the same time its potential significance warrants a careful and continued examination of the data which motivates the confrontation. In this regard, the recent reanalysis of globular cluster age estimates based in part on new parallax measurements obtained from the Hipparcos satellite is very significant.

Globular Cluster ages are obtained by fitting the observed color-magnitude diagram to the predicted distribution for a system of stars of different masses which all form at the same time and which are then evolved on a computer to a certain age. A number of different fitting techniques are employed, with different uncertainties. Because theoretical models predict the instrinsic stellar luminosity, while measurements yield an apparent magnitude, in order to compare theory and prediction, the distance to the globular cluster must be known. Using an extensive Monte Carlo analysis approach begun several years ago[5 it was demonstrated 
that it is precisely this distance determination which leads to the dominant uncertainty in the inferred age of the oldest globular clusters. Based on the distance estimators then available, a $95 \%$ lower limit of 12.1 Gyr was determined for the mean age of 17 of the oldest globular clusters. By comparison, the age of a flat-matter dominated universe with Hubble constant $H_{0}=100 h \mathrm{~km} \mathrm{~s}^{-1} \mathrm{Mpc}^{-1}$, is 6.51[80/h] Gyr, The difference between the lower and upper limits quantified the extent of the cosmic "age problem". This analysis was based on normalizing the color-magnitude diagram by utilizing the inferred instrinsic magnitude of RR-Lyrae stars at a mean metallicity of $[\mathrm{Fe} / \mathrm{H}]=-1.9$ to fix the magnitude of the horizontal branch for the globular clusters under study. By comparing the difference between this magnitude, and the magnitude of the observed main-sequence turnoff point for these clusters to the predicted magnitude difference based on stellar evolution modelling, one infers the age of the clusters. Other analyses obtained similar limits (i.e. see [6]).

Recently, following Hipparcos parallax measurements both for Cepheid variable stars and for various subdwarf main sequence stars (i.e. [7, 8]), we have been prompted to reanalyze the various results on the distance scale to globular clusters, and the resulting age estimates [9]. The changes have been dramatic. A systematic shift in the estimated distances to globular clusters has made it clear that the earlier apparent convergence of such estimates was fortuitous, and the distance data was, and still remains, dominated by systematic errors. Our new best fit age is slightly lower than our previous $95 \%$ lower limit, and the lower limit we obtained (by accounting for the now-apparent systematic uncertainties in the RR Lyrae distance estimators) is 9.6 Gyr. Even allowing for minimum time of 0.2 Gyr after the BIg Bang for the galaxy to form, this new lower limit is consistent with a flat matter dominated universe Hubble age if $H_{0} \leq 66$.

While this might suggest that the need to consider a cosmological constant has now vanished, it is important to remember that the age problem was just one, albeit an important one, of several cosmological arguments which together pointed in this direction. Thus one must combine this new age estimate with the other constraints, which themselves have evolved, in order to reanalyze this issue. Following [2], I display in Figure 1a, for a flat Universe with cosmological constant $\Lambda$, the parameter space of $h$, vs $\Omega_{0}=\rho_{\text {matter }} / \rho_{\text {crit }}$ 
(where $\rho$ is density and $\rho_{\text {crit }}$ is the critical density for a flat universe, so that $\Omega_{\Lambda}=1-\Omega_{0}$ ), showing the allowed region given new age constraint, $15 \geq \tau \geq 9.8$ Gyr, along with the other new constraints I shall describe momentarily. For reasons which will also become clear shortly, I present in Figure 1b, the allowed range in the same parameter space for an open universe.

The quoted upper limit of 15 Gyr is obtained by taking the $95 \%$ upper limit of $\approx 14$ Gyr obtained from the analysis in [9] and adding 1 Gyr as an upper limit to the estimated time after the big bang before star formation began in what would become the halo of our galaxy. Based on recent observations of primeval galaxies at redshifts in excess of 3 , this seems a reasonable upper limit on the time before structures began to form.

The other constraints displayed in figure 1 come from a consideration of the two other independent fundamental sets of cosmological observables, the baryon content of the universe, and large scale structure. I shall describe each in turn below. First, however, it is worth pointing out that current estimates of the Hubble Constant have themselves evolved. Two years ago, there was apparent incompatibility between HST measurements based on Cepheid distances to Virgo, and those based on Supernova Type 1a distance measurements. These two distance measures have been converging (i.e. [10]), so that now a range for $H_{0}$ of $\approx 65 \pm 13$ brackets both measurements. The horizontal dashed lines in the figures display this presently preferred suggested range for $H_{0}$.

For over 20 years a robust upper limit on the baryon density of the universe has come from considerations of Big Bang Nucleosynthesis (BBN). The predicted primordial abundance of the light elements up to ${ }^{4} \mathrm{He}$, all of which are known to be produced primarily during $\mathrm{BBN}$ and not in stars, is a function in each case of the baryon to photon ratio in the universe. Hence a detailed comparison of all the inferred primordial light element abundances with predicted abundances yields a restricted allowed range of baryon densities. That is, of course if there is concordance.

During the past two years there has been a great deal of heat, and some light, shed in this area. First, starting about two years ago, BBN predictions [11] began to tighten the allowed range of $\Omega_{B}$. It began to become clear that unless systematic uncertainties in ${ }^{4} H e$ 
were allowed for the upper limit on $\Omega_{B}$ coming from ${ }^{4} \mathrm{He}$ alone was becoming dangerously close to the lower limit.

The most robust upper limit on $\Omega_{B}$, however has for over 20 years come from observations of deuterium. Deuterium is only destroyed in stellar processing, so any observation of the interstellar or solar system abundance would put a lower bound on the actual primordial abundance of deuterium. Since the predicted deuterium BBN remnant abundance is a monotonically falling function of $\Omega_{B}$, a lower bound on deuterium translates into an upper bound on $\Omega_{B}$. The observed instellar value of $D / H \geq(1.6 \pm 0.2) \times 10^{-5}$ puts a limit $\Omega_{B} h^{2} \leq .027$

This situation took on a new dimension two years ago with the first claimed observation of deuterium absorption lines in primordial hydrogen clouds illuminated by distant quasars [12]. This method in principle allows a direct determination of the primordial deuterium abundance, and hence a direct measure of $\Omega_{B}$. The only problem is that shortly after the original measurement, which gave an anomalously high value for the primordial deuterium abundance, other observations gave a value almost one order of magnitude lower. This value, $D / H=(2.4 \pm 0.3 \pm 0.3) \times 10^{-5}[13$, 14], is almost equal to the lower limit quoted above, and suggests not only that $\Omega_{B}$ is near its upper limit, but also that there has been little chemical evolution of ${ }^{2} H$. Moreover, it reinforces the requirement that their be systematic errors in the ${ }^{4} \mathrm{He}$ abundance estimates if there is to be concordance in BBN. [15, 16, 17].

Most recently, the original high deuterium observation has been withdrawn. However, at the present time, until more observations are made, it seems premature to require that $\Omega_{B}$ is near its upper limit. We have seen over and over again that systematic errors are the dominant contribution to our uncertainty in cosmological quantities, and thus large shifts within the allowed range are as likely as small shifts. Moreover, concordence between the deuterium estimates and the ${ }^{4} \mathrm{He}$ estimates requires some significant systematic error in one of these values. Nevertheless, it is reassuring that even with the new data, a robust estimate of allowed range of $0.01 \leq \Omega_{B} h^{2} \leq 0.0265$ [15] from BBN, allowing for maximal systematic uncertainties in all abundance estimates remains compatible with the data. This range is slightly larger than another independent estimate [16] but is more easily compatible with 
the new deuterium abundance observations.

Even this relatively large upper bound on $\Omega_{B} h^{2}$ is clearly incompatible with a flat baryon dominated for any reasonable Hubble Constant. What is more surprising, however, is that it seems incompatible with a flat universe at all unless the Hubble constant is extremely small, or there remains a large unclustered component of the energy density of the universe. This result arises from considerations of X-Ray measurements of hot gas in rich galaxy clusters. If the gas in these systems is in hydrostatic equilibrium, and the overall gravitational potential is relativity smooth, one can invert an X-Ray temperature/luminosity profile to get both the mass in hot gas, which is by far the largest baryonic component to the mass of the cluster, and also the total mass of the cluster. Thus, one gets a direct estimate for $f_{B}=\Omega_{B} / \Omega_{0}$, where $\Omega_{0}$ represents the fraction of the closure density which is in the form of clustered mass in the universe. If one assumes, for example, that $\Omega=1$, and non-relativistic matter dominates, then $\Omega_{0}=\Omega=1$.

In 1993, White et al [18] argued that the estimate of $f_{B}$ obtained from the Coma cluster was sufficiently large so that it would be incompatible with the BBN upper limit for a flat matter dominated universe unless the Hubble constant were extremely small. Since that time a number of analyses of a broader class of clusters confirms the large $f_{B}$ estimates [19, 20], and these were utilized in previous work to put constraints in $\Omega_{0}$ and $h$ space [2, 3]. Recently, a comprehensive theoretical analysis of cluster modeling has been completed 21] to explore the robustness of the X-Ray interpretations. There is remarkable consistency between the numerical profiles and the data for a value of $\left.f_{B}=0.060 \pm .003\right) h^{-3 / 2}$. A comparison of this value with the BBN constraint would put very severe limits on $\Omega_{0}$. However, if one desires a more conservative upper limit on $\Omega_{0}$ one should consider the lowest possible value of $f_{B}$ which would be consistent with the data. This value assumes all baryons are in the form of hot gas in the cluster and is given by $0.043 h^{-3 / 2}$ [21]. Similarly, taking the largest value of $f_{B}$ consistent with all the data, and allowing for a baryon fraction as large as .013 from stars and dark baryonic halo objects yields an approximate upper limit $0.078 h^{-3 / 2}$ for the range of $h$ values of interest here.

Combining these limits with the BBN bounds yields a conservative constraint $0.12 \leq$ 
$\Omega_{0} h^{1 / 2} \leq 0.60$. This is displayed in Fig 1 for both an open and flat universe with cosmological constant.

Finally, the last set of constraints comes from considerations of large scale structure. This is another area where progress has been made recently, in particular as a result of measurements of CMB anisotropies. For some time, various independent observations have suggested that while the clustered mass in the universe exceeds the upper limit on the baryon density coming from BBN, it nevertheless falls short of the closure density. Recent observations confirm this trend. Nevertheless, virial estimates suggest that $\Omega_{0} \geq 0.3$, and I adopt this conservative lower bound here, as displayed in Fig 1.

I shall utilize two additional large scale structure constraints in this analysis. First, observations of galaxy correlations supply a constraint on the shape of the power spectrum of density fluctuations on large scales. This is an extremely powerful constraint because it is related to the primordial power spectrum primarily by considerations of causality, and the density of clustered matter in the universe. While the result is somewhat model dependent, assuming a Cold Dark Matter dominated universe and assuming a roughly scale invariant initial spectrum of density perturbations, as predicted by inflation and as allowed by COBE constraints on the CMB, Peacock and Dodds [22] obtained the constraint $\Gamma=\Omega_{0} h \exp \left(-\Omega_{B}-\Omega_{B} / \Omega_{0}\right)=0.255_{-.033}^{+.038}$, which has been used in previous analyses. More recently both these authors [23, 24] have noted that non-linear effects might alter their results on short scales. Removing the shortest scale points and refitting one obtains [25, 26] $\Gamma=0.230_{-.034}^{+.042}+0.28(1 / n-1)$ at the 95 per cent confidence level. Here $\mathrm{n}$ is the spectral index of the primordial density perturbations, which CMB observations suggest is between $\approx 0.9-1.1$. We display this constraint in both Figure 1a and $1 \mathrm{~b}$, as it is insensitive to the presence or absence of a cosmological constant.

Finally, recently a number of authors [27, 28, 29] have examined the abundance of galaxy clusters, which constrains the magnitude of density fluctuations on intermediate scales. By comparing to the COBE normalized value on large scales [30], one can put a constraint on $\Omega_{0}$ vs $h$ which is complementary to the shape constraint described above, although it turns out to be provide limits which are quite similar. Because the growth of fluctuations between 
COBE scales and galaxy cluster scales is dependent on the geometry of the universe, this constraint is slightly different for open vs flat cosmological constant dominated cosmologies. We plot these constraints [25, 26] in Figures 1a and 1b.

The interplay between all of these new constraints in the $\Omega_{0}$ vs $h$ parameter space is quite significant. Most important, by shifting the allowed region in $h$ upward, the new age limit combines with the other constraints so that now a larger region of parameter space is allowed for an open universe than for a flat universe with cosmological constant. In particular, it is now clear that smaller values of a Hubble constant yield a universe which is now too old in a cosmological constant dominated universe. The significance of the shift in age estimate which has taken place can be seen in both figures, where a solid curve displays what used to the upper limit on the allowed phase space coming from the earlier age constraint. For an open universe, this old limit was extremely constraining. In this regard, the recent decrease in estimates for the Hubble constant coming from HST measurements is also relevant.

It is also clear that while globular cluster age estimates have relaxed the constraint on the overall matter density of the universe, the other updated cosmological constraints coming from large scale structure and baryon counting have solidified in a region in which a flat, matter dominated universe is marginally viable only for an extremely small Hubble Constant.

The debate thus appears to remain between an open universe and flat universe with cosmological constant. On the basis of shear size of allowed parameter space, for the first time an open universe is more strongly favored. There are, however, additional theoretical and observational data which bear on this conclusion. First, several preliminary observations have been made which are claimed to directly constrain $\Omega_{0}$ and $\Lambda$ which on balance may swing slightly in favor of a non-zero cosmological constant. Cluster evolution, in number density [31], and luminosity density [32], has recently been used to place constraints on both $\Omega_{0}$, and $\Lambda$. The former, also displayed in figures $1 \mathrm{a}$ and $1 \mathrm{~b}$, yields a bound on $\Omega_{0}$ which is inconsistent with a flat universe, but reasonably independent of the presence or absence of a cosmological constant. The latter however, is claimed to strongly favor a non-zero cosmological constant. In fact, a lower bound on $\Omega_{\Lambda}$ of 0.37 at the $99 \%$ confidence level is claimed. This result is displayed in figure 1a, but it should be taken as preliminary. 
To counterbalance it, recent observations of the luminosity-redshift relation for type 1a supernovae [33] place a preliminary upper limit on the value of $\Omega_{\Lambda}$ of 0.49 . While this result involves the first application of a new technique, the recent measurements of more high redshift supernovae should, within the next few years, provide the strongest constraints on a cosmological constant in the universe today.

Eventual measurements of cosmic microwave anisotropies on small angular scales will allow a direct measurement of the cosmological constant, in addition to other fundamental cosmological parameters, at the 10-20\% level (i.e. [34]). A distinction can be made between the effects of a cosmological constant, and a non zero curvature. For a flat universe, increasing $\lambda$, which implies decreasing the ratio $\Omega_{0} / \Omega_{B}$, causes the magnitude of the first Doppler peak to increase, while its position is relatively unaffected. An open universe, on the other hand, changes the position of the first doppler peak, because introducing a non-zero curvature alters the redshift-angular scale relation of the universe. In this regard, present measurements of CMB anisotropies suggest a large doppler peak in the location expected for a flat universe. Thus, while extremely preliminary, CMB measurements on small angular scales may favor of a cosmological constant over an open universe [35].

At present, it is clearly too early to choose one cosmological model over the other. It is clearly getting increasingly difficult to find accord with a flat universe without a cosmological constant. The question then becomes: Which fundamental fine tuning problem is one more willing to worry about: the flatness problem, or the cosmological constant problem? The latter involves a fine tuning of almost 125 orders of magnitude, if the cosmological constant is non-zero and comparable to the density of clustered matter today, while the former involves a fine tuning of perhaps only 60 orders of magnitude if one arbitarily fixes the energy density of the universe at the planck time to be slightly less than the closure density. Numerological arguments might thus suggest that one should be more prepared to give up flatness than a zero cosmological constant. I claim however, that this argument is incomplete.

We have a perfectly good theory, involving physics well below the planck scale, for why we might live in a flat universe. As long as there was an inflationary regime in the early universe, the universe generically is driven to be approximately flat to many more decimal 
places than are required to resolve the flatness problem. Moreover, when considering possible particle physics models of the early universe, inflation seems to be ubiquitous. The difficulty seems to be not how to get enough inflation, but rather how to end it. On the other hand, we have absolutely no theory of the cosmological constant at all. Other than vague a priori prejudice, there is no well defined physical argument at the present time suggesting a zero, rather than arbitrarily small value of this quantity. Moreover, the energy scale associated with a non-zero cosmological constant which dominates the universe today is not unusual. It corresponds to the characteristic mass scale which is discussed for neutrino masses which

might solve the solar neutrino problem. Also interesting, recent arguments suggest that if the laws of physics predict a distribution of universes, with randomly chosen values for the cosmological constant, then quantitative anthropic arguments make it not implausible that it should be observed to be comparable to the matter density in the universe today [36]. Whatever one's views toward anthropic arguments, it is not clear that the same reasoning could be applied to the flatness problem. Precisely because we have physical laws which suggest the universe should be flat, I would argue the a priori probability distribution for the curvature parameter one might reasonably consider should be strongly peaked about zero, in which case anthropic arguments along the lines applied to the cosmological constant might not be as suggestive, to the extent such arguments are suggestive.

In conclusion, we should know within a decade whether the cosmological constant is nonzero, and whether we live in a flat universe. The recent resolution of the age problem has dramatically altered the case for a cosmological constant vs an open universe. A combination of cosmological observations now allows a larger parameter space for an open universe than a flat universe with cosmological constant. While one might argue that theoretical prejudice still favors the latter, if either of these cases represents reality, the implications for fundamental particle physics will be profound.

\section{Acknowledgments}

This work was supported in part by the DOE, and Case Western Reserve University. I wish to thank CERN for a stimulating environment while this work was completed, my 
collaborators on globular cluster age estimation, Brian Chaboyer, Pierre Demarque, and Peter Kernan, and Steve Weinberg for enlightening discussions on anthropic arguments in favor of a cosmological constant.

\section{References}

[1] see e.g., M.S. Turner, G. Steigman, and L. Krauss, Phys. Rev. Lett. 52, 2090 (1984); M.S. Turner, Physica Scripta T36, 167 (1991); P.J.E. Peebles, Astrophys. J. 284, 439 (1984); G. Efstathiou et al., Nature 348, 705 (1990); L. A. Kofman and A.A. Starobinskii, Sov. Astron. Lett. 11, 271 (1985); L. A. Kofman, N.Y.Gnedin, and N.A. Bahcall, Astrophys. J. 413, 1 (1993).

[2] L.M. Krauss and M.S. Turner, Gen. Rel. Grav. 27, 1137 (1995)

[3] J. P. Ostriker, P. Steinhardt, Nature 377, 600 (1995)

[4] S. Weinberg, Rev. Mod. Phys. 61, 1 (1989).

[5] B. Chaboyer, P. Demarque, P. J. Kernan, L.M. Krauss, Science 271, 957 (1996)

[6] M. Bolte, C. Hogan, Nature 3761995

[7] M.W.Feast, \& R.W.Catchpole, Mon. Not. R. Astron. Soc., 286, L1 (1997)

[8] I.N .Reid, astro-ph/9704078, Astrophys. J., in press

[9] B. Chaboyer, P. Demarque, P. J. Kernan, L.M. Krauss, astro-ph/9706128, submitted to Astrophys. J.

[10] W. L. Freedman, astro-ph/9612024, to appear Proceedings for "Critical Dialogs in Cosmology", , Princeton 250th anniversary ed. N. Turok

[11] P.J. Kernan and L.M. Krauss, Phys. Rev. Lett, 72. 3309 (1994).

[12] A. Songaila, L.L. Cowie, C. Hogan, and M. Rugers, Nature 368, 599 (1994) 
[13] D. Tytler, X.M. Fan, and S. Burles, Nature 381, 207 (1996)

[14] D. Tytler, S. Burles, and D. Kirkman, astro-ph/9612121

[15] L.M. Krauss and P. J. Kernan, Phys. Lett. B, 347, 347 (1995).

[16] C. Copi, D.N. Schramm, and M.S. Turner, Science 267, 192 (1995).

[17] N. Hata et al, Phys. Rev. Lett. 75, 3977 (1995)

[18] S.D.M. White et al., Nature 366, 429 (1993)

[19] D.A. White and A.C. Fabian, Mon. Not. R. Astron. Soc., 273, 72 (1995)

[20] J.P. Henry, U.G. Briel, and P.E.J. Nulsen, Astron. Astrophys. 271, 413 (1993)

[21] A.E. Evrard, C. A. Metzler, and J.F. Navaro, Astrophys. J. 469, 494 (1996); A.E.Evrard,Mon. Not. R. Astron. Soc., in press, astro-ph/9701148 (1997)

[22] J.A. Peacock and S.J. Dodds, Mon. Not. R. astr. Soc. 267, 1020 (1994).

[23] J.A. Peacock, astro-ph/9601135 (1996)

[24] J.A. Peacock and S.J. Dodds, astro-ph/9603031 (1996)

[25] A.R. Liddle, D.H.Lyth, P.T.P.Viana, M. White, Mon. Not. R. astr. Soc. 282, 281 (1996)

[26] A.R. Liddle, D.H.Lyth, D. Roberts, P.T.P.Viana, Mon. Not. R. astr. Soc. 278, 644 (1996)

[27] P.T.P. Viana, A.R. Liddle, Mon. Not. R. astr. Soc. 281, 323 (1996)

[28] A.R. Liddle, P.T.P. Viana, astro-ph/9610215

[29] S.D.M. White, G. Efstathiou, C.S. Frenk, Mon. Not. R. astr. Soc. 262, 1023 (1993)

[30] M. White, E.F. Bunn, Astrophys.J., 450, 477 (1995) 
[31] N.A. Bahcall, X. Fan, and R. Cen, astro-ph/9706018

[32] T. Totani, Y. Yoshii, and K. Sato, astro-ph/9705014

[33] S. Perlmutter et al, astro-ph/9602122

[34] A.Kosowsky, M. Kamionkowski, G. Jungman, D. Spergel astro-ph/9605147, Nucl.Phys.Proc.Suppl. 51B, 49 (1996)

[35] L.M. Krauss, D. Scott, J. Silk, M. White, to appear.

[36] H. Martel, P.R. Shapiro, S. Weinberg, astro-ph/9701099 (1997) 


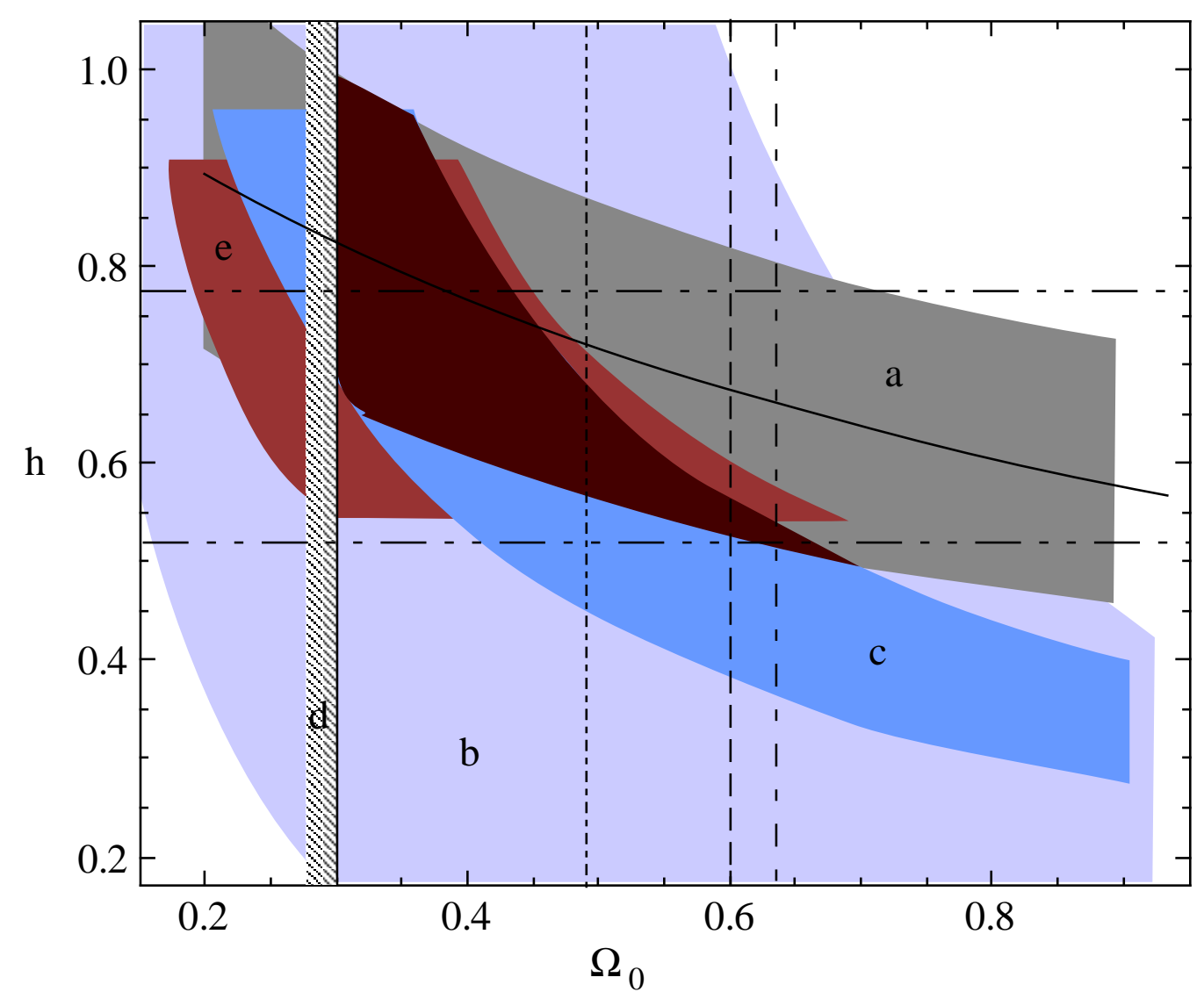

Figure 1: Shaded regions represent constraints on $h$ vs. $\Omega_{0}$ for a flat universe with cosmological constant arising from (a) globular cluster age limit, (b) baryon content of the universe, (c)shape of galaxy power spectrum, (d) lower limit on clustered mass from virial estimates, (e) abundance of galaxy clusters extrapolating from COBE normalization, assuming dark matter is cold. Shown in dark shading is the locus of points in phase space satisfying all limits. Horizontal dashed lines present the upper and lower limits of the present preferred range of the Hubble constant quoted in the text. The dashed vertical lines represent various preliminary limits on $\Omega_{0}$ for a flat universe with cosmological constant. The right-most limit represents the claimed $99 \%$ lower limit on $\Omega_{\Lambda}=0.34$ arising from considerations of the evolution of the cluster luminosity function. The next largest limit represents an upper limit on $\Omega_{0}$ from considerations of the evolution of the galaxy cluster number density. The left most limit arises from a claimed upper limit on $\Omega_{\Lambda}$ coming from a measurement of $q_{0}$ using Type 1a supernovae. Finally, the solid curved line represents the previous upper limit on $h$ vs $\Omega_{0}$ using the claimed lower limit on the age of the Universe of 12.1 Gyr which has now been revised downward. 


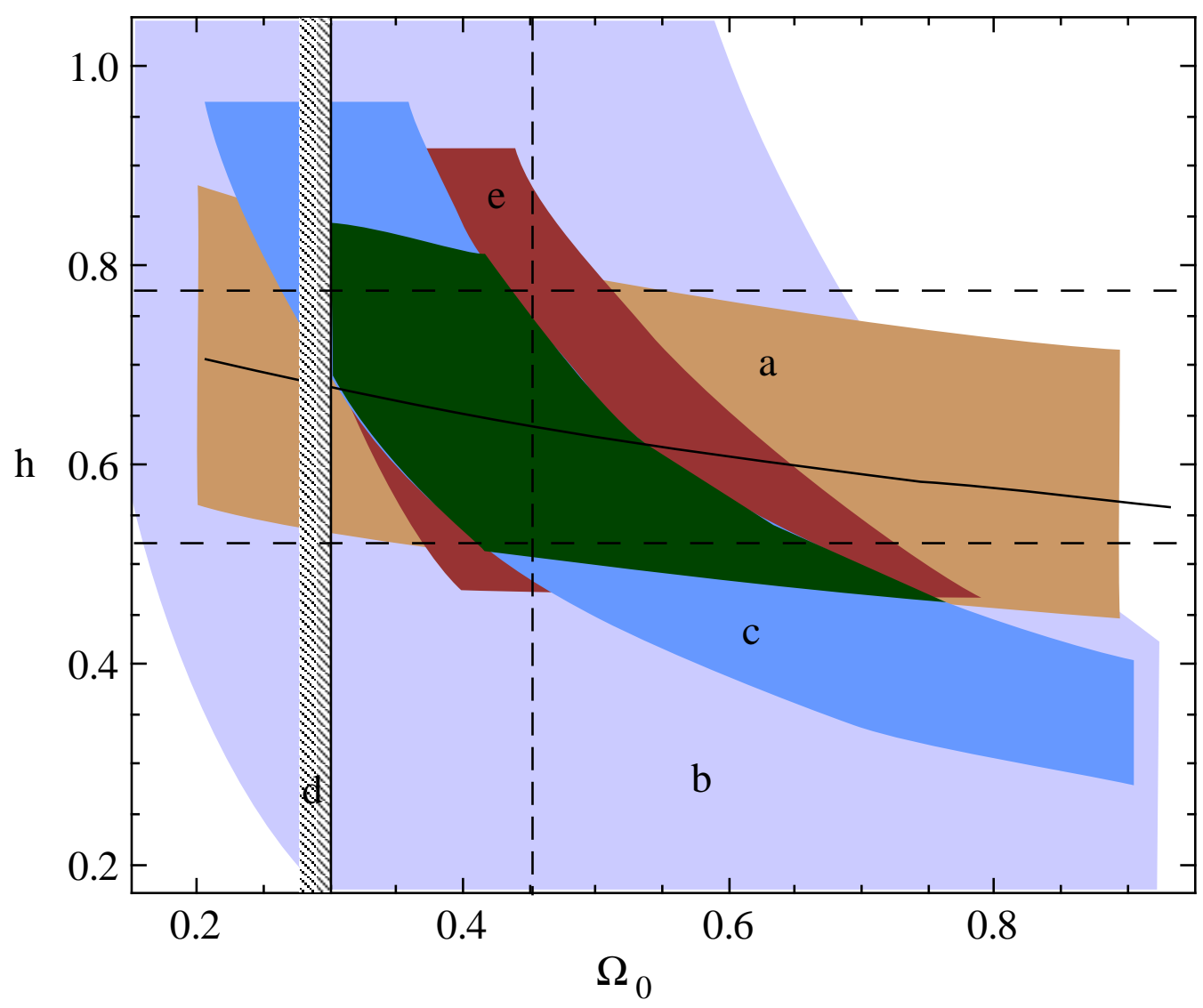

Figure 2: Same as figure 1a, but in this case for an Open Universe. The vertical dashed line represents the claimed upper limit on $\Omega_{0}$ arising from considerations of the evolution of the galaxy cluster number density. 